\title{
Candidemia from an Upper Urinary Tract Infection Complicated by Candida Endophthalmitis
}

\author{
Reina Suzuki ${ }^{1}$, Hitoshi Kuroda ${ }^{1,2}$, Hiroshi Matsubayashi ${ }^{1}$, Akira Ishii ${ }^{1}$, Fumihiko Toyoda ${ }^{3}$, \\ Alan Kawarai Lefor ${ }^{4}$ and Hitoshi Sugawara ${ }^{1}$
}

\begin{abstract}
A 51-year-old Japanese woman developed candidemia as an outpatient secondary to a Candida albicans upper urinary tract infection complicated by previously undiagnosed type 2 diabetes mellitus with poor glycemic control and ureterolithiasis. The patient did not have any risk factors typically associated with candidemia, such as an indwelling vascular catheter, parenteral nutrition or broad-spectrum antibiotic use. During the clinical course, her condition was complicated by unilateral candida endophthalmitis, which progressed despite the administration of systemic antifungal agents and ultimately required vitreous surgery. The etiology of candidemia in this patient and the reason she developed progressive ocular symptoms after starting antifungal treatment are reviewed.
\end{abstract}

Key words: candidemia, Candida albicans, type 2 diabetes mellitus, endophthalmitis

(Intern Med 54: 2693-2698, 2015)

(DOI: 10.2169/internalmedicine.54.4691)

\section{Introduction}

Candidemia is a well-known complication that typically occurs in the patients in the intensive care unit or immunocompromised patients and is associated with various risk factors, such as broad-spectrum antibiotic use, an indwelling vascular catheter, receiving parenteral nutrition, neutropenia or immunosuppressive drug administration (1). It has rarely been reported in an otherwise healthy outpatient. We herein present a 51-year-old woman with previously undiagnosed type 2 diabetes mellitus with poor glycemic control, which is a risk factor for the development of invasive candidiasis, who developed candidemia as an outpatient. Understanding the pathophysiology of candidemia in this patient is important to enable primary care physicians to recognize and manage this serious condition which may otherwise not be considered in the differential diagnosis when treating outpatients.

\section{Case Report}

A 51-year-old woman was hospitalized with a fever, chills, and bilateral flank pain. The patient had been in her usual state of health until she developed right-sided abdominal pain one month prior to admission. Abdominal ultrasound performed by her family physician showed a left ureteral stone measuring $9 \times 9 \mathrm{~mm}$. However, she was not treated at that time. Three days before admission, she developed left-sided abdominal pain associated with a fever. The following day she recorded a temperature of $40^{\circ} \mathrm{C}$, for which she visited another local clinic and oral levofloxacin $500 \mathrm{mg}$ daily was prescribed for a suspected bacterial infection. One day before admission, she visited her family physician with a persistent fever. Blood tests revealed a white blood cell count of $13,500 / \mu \mathrm{L}$ and $\mathrm{C}$-reactive protein concentration of $12 \mathrm{mg} / \mathrm{dL}$. Ceftriaxone was given intravenously for a suspected infection and she returned home.

On the day of admission, the patient developed nausea and vomiting, and an abdominal ultrasound study revealed

\footnotetext{
${ }^{1}$ Division of General Medicine, Department of Comprehensive Medicine 1, Saitama Medical Center, Jichi Medical University, Japan, ${ }^{2}$ Department of Education and Support for Regional Medicine, Tohoku University Hospital, Japan, ${ }^{3}$ Divisions of Ophthalmology, Department of Comprehensive Medicine 2, Saitama Medical Center, Jichi Medical University, Japan and ${ }^{4}$ Department of Surgery, Jichi Medical University, Japan Received for publication December 9, 2014; Accepted for publication February 15, 2015 Correspondence to Dr. Hitoshi Sugawara, hsmdfacp@omiya.jichi.ac.jp
} 
Table. Laboratory Studies at the Time of Admission.

\begin{tabular}{|c|c|c|c|c|c|c|c|c|}
\hline \multirow{2}{*}{$\begin{array}{l}\text { Urinalysis: } \\
\mathrm{pH}\end{array}$} & \multirow[b]{2}{*}{5.0} & & \multicolumn{3}{|c|}{ Serum chemistry tests: } & \multirow[b]{2}{*}{ LDL-C } & \multirow{2}{*}{\multicolumn{2}{|c|}{$162 \mathrm{mg} / \mathrm{dL}$}} \\
\hline & & & $\mathrm{TP}$ & 7.2 & $\mathrm{~g} / \mathrm{dL}$ & & & \\
\hline Specific Gravity & 1.025 & & Alb & 2.8 & $\mathrm{~g} / \mathrm{dL}$ & TG & 304 & $\mathrm{mg} / \mathrm{dL}$ \\
\hline Protein & $2+$ & & AST & 20 & $\mathrm{U} / \mathrm{L}$ & PCT & $>=10$ & \\
\hline Sugar & $4+$ & & ALT & 22 & $\mathrm{U} / \mathrm{L}$ & & & \\
\hline Acetone & $2+$ & & $\mathrm{LDH}$ & 249 & $\mathrm{U} / \mathrm{L}$ & & & \\
\hline Bilirubin & - & & CK & 256 & $\mathrm{U} / \mathrm{L}$ & & & \\
\hline Urobilinogen & $+/-$ & & ALP & 470 & $\mathrm{U} / \mathrm{L}$ & & & \\
\hline Occult blood & $3+$ & & CRP & 31.75 & $\mathrm{mg} / \mathrm{dL}$ & & & \\
\hline CBC: & & & $\mathrm{Na}$ & 129 & $\mathrm{mmol} / \mathrm{L}$ & & & \\
\hline White Blood Cells & 13,540 & $/ \mu \mathrm{L}$ & $\mathrm{K}$ & 4.0 & $\mathrm{mmol} / \mathrm{L}$ & & & \\
\hline Neutrophils & 94 & $\%$ & $\mathrm{Cl}$ & 92 & $\mathrm{mmol} / \mathrm{L}$ & & & \\
\hline Lymphocytes & 3.0 & $\%$ & $\mathrm{Ca}$ & 8.8 & $\mathrm{mg} / \mathrm{dL}$ & & & \\
\hline Monocytes & 3.0 & $\%$ & $\mathrm{P}$ & 2.5 & $\mathrm{pg} / \mathrm{mL}$ & & & \\
\hline Red Blood Cells & $465 \times 10^{4}$ & $/ \mu \mathrm{L}$ & BUN & 26 & $\mathrm{mg} / \mathrm{dL}$ & & & \\
\hline Hemoglobin & 13.9 & $\mathrm{~g} / \mathrm{dL}$ & $\mathrm{Cr}$ & 1.25 & $\mathrm{mg} / \mathrm{dL}$ & & & \\
\hline Platelets & $19.8 \times 10^{4}$ & $/ \mu \mathrm{L}$ & UA & 8.0 & $\mathrm{mg} / \mathrm{dL}$ & & & \\
\hline & & & HDL-C & 39 & $\mathrm{mg} / \mathrm{dL}$ & & & \\
\hline
\end{tabular}

CBC: Complete Blood Count, TP: Total Protein, Alb: Albumin, AST: Asparate Aminotransferase, ALT: Alanin Aminotransferase, LDH: Lactate Dehydrogenase, CK: Creatine Kinase, ALP: Alkaline Phosphatase, CRP: C - Reactive Protein, BUN: Blood Urine Nitrogen, UA: Uric Acid, HDL-C: High Density Lipoprotein Cholesterol, LDL-C: Low Density Lipoprotein Cholesterol, TG: Triglyceride, PCT: Procalcitonin

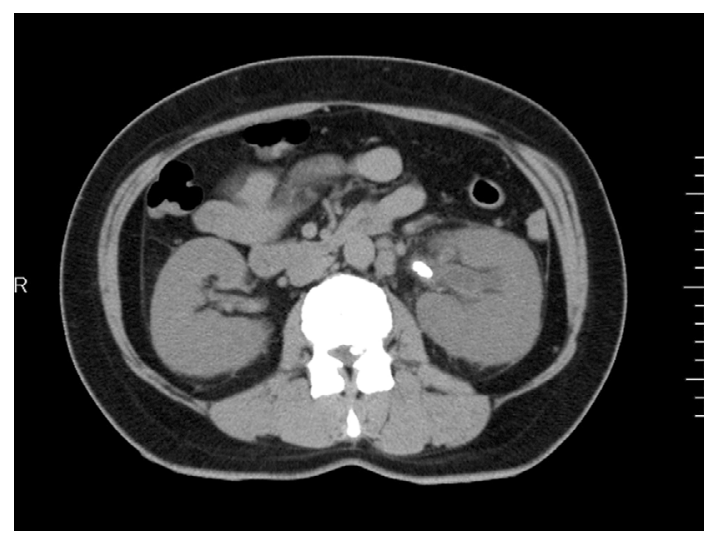

Figure 1. Plain abdominal computed tomography. A stone in the left ureter resulted in hydronephrosis of the left kidney.

left hydronephrosis. The HbA1c [National Glycohemoglobin Standardization Program (NGSP)] was $11 \%$ from the previous day. An upper urinary tract infection (UTI) complicated by a ureteral stone and diabetes mellitus was diagnosed and she was referred for further evaluation after a single dose of ampicillin/sulbactam ( $3 \mathrm{~g}$ ) was given intravenously. Neither a urinalysis nor a urinary gram stain was performed at that time.

On this admission, the patient reported nausea, vomiting, diaphoresis, urinary frequency, loss of appetite and involuntary weight loss of $2 \mathrm{~kg}$ over the previous four months. She denied a cough, sputum production, neck stiffness, pain on urination, or visual disturbances. Her past medical history was notable only for cystitis at 31 years of age, and the most recent medical evaluation three years previously showed no abnormalities. She had no prior hospitalizations or surgery. She was not taking any over-the-counter medicines and she had no known allergies. There was no family history of malignancy or diabetes. She was married and lived with her husband and son. She worked as an instructor in the art of flower arranging. She denied smoking, drinking alcohol or illicit drug use.

On the physical examination, the patient was alert and in no acute distress. The patient was $167 \mathrm{~cm}$ tall and weighed $75 \mathrm{~kg}$, with a body mass index of $27 \mathrm{~kg} / \mathrm{m}^{2}$. Her temperature on admission was $37.7^{\circ} \mathrm{C}$, blood pressure $116 / 87$ $\mathrm{mmHg}$, pulse $136 / \mathrm{min}$ and regular, respiratory rate $16 / \mathrm{min}$ and oxygen saturation of $97 \%$ on room air. Her left conjunctiva was slightly hyperemic. There were no murmurs on cardiac auscultation. There was no tenderness on abdominal palpation, however, percussion tenderness at the costovertebral angle was present bilaterally. Furthermore, there was edema of the extremities. The remainder of the physical exam was unremarkable.

The laboratory studies showed (Table), hypoalbuminemia, hyponatremia, elevated white blood cell count, lactate dehydrogenase, alkaliphosphatase, $\gamma$-glutamyl transpeptidase, Creactive protein, blood urea nitrogen, creatinine, uric acid, low-density lipoprotein cholesterol, triglycerides, serum (random) glucose and HbA1c. The urinalysis revealed aciduria and was positive for glucose, occult blood, protein and ketone bodies, and negative for nitrates. The result of the urine gram stain was not available on this admission but was later found to be negative for yeast-like fungus. The serum glucose level was $359 \mathrm{mg} / \mathrm{dL}$, which fulfilled the diagnostic criteria for type 2 diabetes mellitus combined with the HbA1c of $11 \%$. An electrocardiogram showed sinus tachycardia (122/min) and slightly decreased ST-T waves in leads V3 and V4. A chest X-ray was normal. An abdominal computed tomography scan without contrast (Fig. 1) revealed a high density lesion measuring $9 \mathrm{~mm}$ in the left ureter associated with dilatation of the left renal pelvis and increased fat density around the left kidney, suggesting inflammation of the left kidney. 

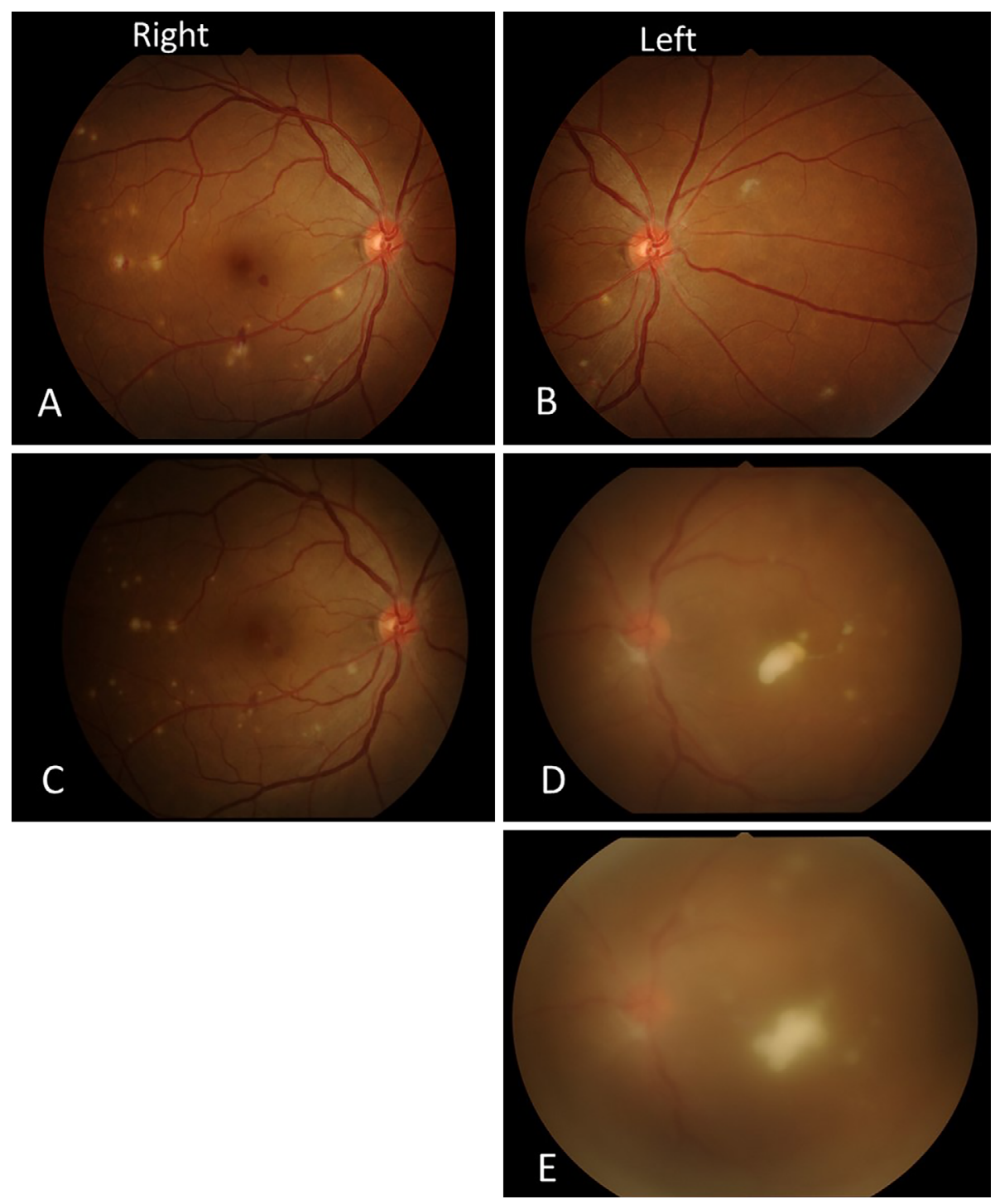

Figure 2. Fundoscopic findings. A, B: White plaques consistent with a fungal infection are seen in both retinas five days after admission. $\mathrm{C}$, D: Inflammation of the left eye is seen extending to the vitreous body, consistent with endophthalmitis, on hospital day 12. E: An exacerbation of the white plaques and opacity of the vitreous body in the left eye on hospital day 18.

The patient was diagnosed with acute pyelonephritis complicated by hydronephrosis of the left kidney in the presence of a ureteral stone and type 2 diabetes mellitus. Ampicillin/ sulbactam ( $3 \mathrm{~g}$ ) was administered every 8 hours along with intravenous fluid administration. Insulin therapy was initiated soon after the blood and urine cultures were obtained.

Despite antimicrobial therapy, the patient's fever persisted and her general condition did not improve. On hospital day five, Candida albicans (C. albicans) was detected in both the blood and urine cultures obtained on admission. Micafungin (100 mg daily) was then added, in addition to ampicillin/sulbactam. The $\beta$-D glucan concentration obtained on the same day was $299 \mathrm{pg} / \mathrm{mL}$ and Candida mannan antigen was positive. A retinal examination showed white plaques consistent with a fungal infection of both retinas (Fig. 2A, B), although the patient reported no visual disturbances. An echocardiogram on the same day demonstrated no signs of candida endocarditis.
A ureteral stent was placed in the left ureter on the seventh hospital day. On the eighth hospital day, micafungin was changed to fosfluconazole (400 mg daily) due to its increased penetration into the intraocular space. This change in treatment was also due to the minimal excretion of micafungin in the urine. Furthermore, there is inadequate evidence supporting its use in the treatment of urinary tract infections (1). C. albicans was detected in the vaginal smears at this time. Following fosfluconazole therapy, the patient's temperature began to decrease. Her ocular symptoms became worse, however, and "floaters" in the left eye associated with decreased vision from 24/20 to 1/20 developed on the eleventh hospital day. Ophthalmologic follow-up on hospital day 12 demonstrated the further exacerbation of inflammation in the left eye extending to the vitreous body, which was consistent with endophthalmitis (Fig. 2C, D). White plaques on the left retina and the vitreous opacity continued to grow (Fig. 2E), and the patient finally under- 


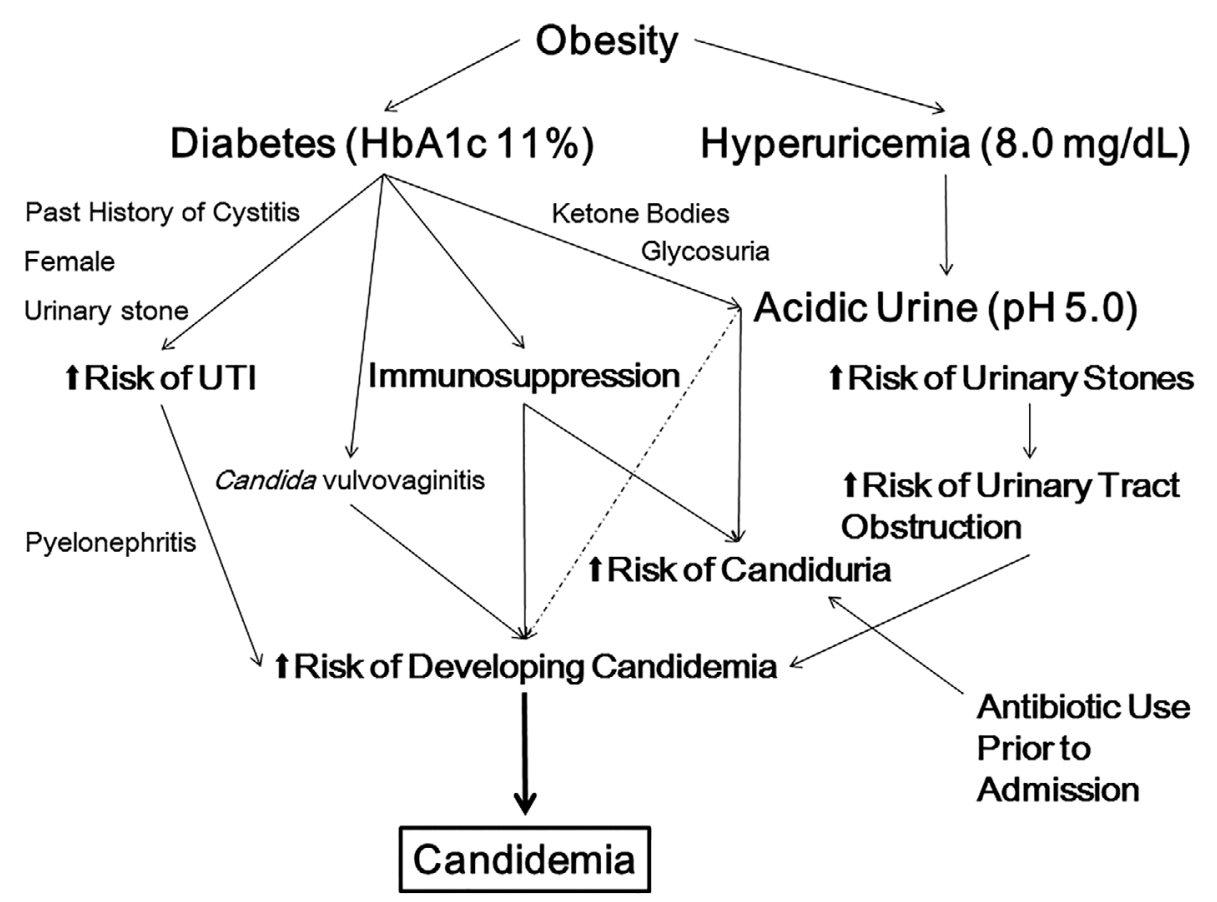

Figure 3. The proposed pathophysiology of candidemia in the present patient.

went vitreous surgery for candida endophthalmitis in the left eye on hospital day 20. The postoperative course was uneventful.

The blood cultures were evaluated weekly, and disappearance of $C$. albicans from the blood stream was confirmed on hospital day 12 . We discontinued the antifungal treatment on hospital day 32, approximately two weeks after the clearance of $C$. albicans from the blood was confirmed. The laboratory studies returned to normal levels on hospital day 35. She was discharged on hospital day 36 on an insulin regimen. During follow-up, she underwent extracorporeal shock wave lithotripsy three times for a left ureteral stone. Her left vision returned to $14 / 20$ three months after discharge.

\section{Discussion}

Two important clinical issues arise from reviewing the clinical course of the present patient. First, candidemia may occur in an outpatient and clinicians must be aware of this because unrecognized candidemia can result in severe complications. Second, the treatment of candida endophthalmitis requires careful attention and regular ophthalmologic followup.

\section{The etiology of candidemia (Fig. 3)}

The working hypothesis for the etiology of candidemia in the present patient is $C$. albicans which colonized the bladder, then migrated retrograde to the left kidney where it caused pyelonephritis, which was facilitated by hydronephrosis due to a pre-existing ureteral stone. This may have offered a point of entry for Candida into the blood. There are three known routes for the development of candidemia, in- cluding an indwelling vascular catheter which offers a direct route of entry, an impaired gastrointestinal tract mucosal barrier due to antibiotic use or neutropenia, and a localized focus of infection, such as pyelonephritis (2). In the present patient, the positive blood and urine cultures for C. albicans strongly suggest candidemia due to a candida upper UTI, combined with unilateral hydronephrosis and a ureteral stone. The absence of other well-known risk factors for the development of invasive candidiasis, such as the presence of an indwelling vascular catheter or administration of parenteral nutrition (1), additionally supports this explanation, although we cannot completely rule out the possibility that short-term antibiotic use prior to admission may have been a factor in this patient. To the best of our knowledge, there is no precise definition for the duration of antibiotic administration which significantly increases the risk of developing candidemia.

A retrograde Candida UTI is not a common clinical presentation and occurs almost exclusively in the patients with diabetes or anatomical abnormalities of the urinary tract, including renal stones causing obstruction $(3,4)$. Before a Candida infection is established, adherence and colonization near the site of infection are critical and it is reported that greater mucosal adherence leads to increased virulence. $C$. albicans poorly adheres to the bladder mucosa; however, under certain circumstances, such as immunosuppression or co-infection with E. coli, colonization can be enhanced (5).

The combination of diabetes and urinary tract obstruction may help Candida to successfully colonize the urinary tract by promoting urinary stasis and, provided that colonization is essential prior to Candida infection, the contribution of these circumstances to the development of candidemia is readily understandable. In the patients with diabetes, the 
presence of glycosuria may also help Candida to grow in the bladder in combination with decreased phagocytic activity.

More recently, Fisher et al. proposed acidic urine to be a possible contributing factor to the increased incidence of Candida UTIs in the patients with poorly controlled diabetes (5) according to the findings of Abaitua et al. that Candida growth was promoted under the conditions of acidic $\mathrm{pH}$ combined with nitrogenous compounds (6). Thus, the significantly acidic urine in the present patient due to glycosuria plus hyperuricemia on admission may have played a role in the development of candidemia. The correlation between acidic urine and a retrograde Candida UTI remains somewhat speculative. However, given that acidic urine is also reported to be a risk factor for uric acid, cystine and calcium oxalate stone formulation (7), a contributing factor for the development of a retrograde infection due to urinary obstruction, there is a possibility that acidic urine may have played a role in the pathophysiology of candidemia in the present patient.

It is crucially important to note that the present patient suffered from uncontrolled and previously undiagnosed diabetes at the time of admission, in the absence of other risk factors for the development of candidemia such as an indwelling vascular catheter. Diabetes is an important underlying disease which predisposes patients to the development of candidemia and is seen in approximately one third of all patients with candidemia $(8,9)$. Diabetes is a risk factor for the development of UTIs (10), candiduria (3), candidemia $(8,9)$ and, most importantly, the progression from candiduria to candidemia as discussed above. Antifungal treatment for candiduria is still controversial, however, it is not routinely recommended because the long-term outcomes are usually benign (11). Candiduria does not generally lead to invasive candidiasis unless there are co-existing conditions. When there is an increased risk for the development of candidemia in the patients with diabetes mellitus, however, close follow-up, including performing a urinalysis and urine gram stain, should be considered, even if they are treated as outpatients. These measures may enable the earlier recognition of acute candidemia, so that appropriate treatment can thus be given in a timely manner.

\section{Candida endophthalmitis}

It is notable that the present patient suffered from progressive ocular symptoms, which ultimately required a surgical procedure to treat Candida endophthalmitis of the left eye while she additionally received intravenous systemic antifungal agents. The definition of endophthalmitis varies in the literature. It is defined as the involvement of the intraocular spaces $(12,13)$, but also as isolated chorioretinitis (14). In the present report, however, we use the term "endophthalmitis" to refer to disease extending to the vitreous body according to the clinical findings. Endogenous fungal endophthalmitis is a critical condition with an increased risk of vision loss and is a relatively frequent complication of candidemia. There are few studies of endogenous fungal endophthalmitis in the current literature and the largest retrospective case series reviewed 51 patients with endogenous fungal endophthalmitis diagnosed with positive vitreous cultures (no mention of blood cultures); the most frequently isolated microorganism was C. albicans in $65 \%$ of the patients. Bilateral involvement was observed in only 14 patients $(27 \%)(13)$.

In the present patient, there were fungal plaques in both eyes initially, however, the disease extended to the vitreous body only in the left eye despite the use of antifungal agents. This suggests two important issues in preventing endophthalmitis: choosing an appropriate antifungal agent with good ocular penetration early in the treatment course and changing to an alternative antifungal agent if the initial therapy is not effective.

In the present patient, micafungin was started on the fifth hospital day, soon after C. albicans was detected from two sets of blood cultures obtained on admission, according to the Infectious Disease Society of America guidelines (1), which recommend micafungin use for moderate candidemia. Therapy was then changed from micafungin to fosfluconazole on hospital day eight without a loading dose due to the elevated levels of biliary enzymes. The decision to use fosfluconazole was made three days after initiating therapy, which may have contributed to the progression of ocular symptoms. Although the patient's vision improved during the postoperative period, she may have benefited from an earlier change to fosfluconazole and with an adequate loading dose.

Regarding the treatment for candida endophthalmitis, the Infectious Disease Society of America guidelines recommend the use of intravenous amphotericin B and oral flucytosine, using fluconazole in patients with less severe infections possibly with earlier surgical intervention (1). These guidelines were established in 2009, possibly before newer antifungal agents became widely available. Riddel et al. referred to this point and proposed an updated therapy for candida endophthalmitis which recommends fluconazole (12 $\mathrm{mg} / \mathrm{kg}$ loading dose, then $6-12 \mathrm{mg} / \mathrm{kg} /$ day), voriconazole (6 $\mathrm{mg} / \mathrm{kg}$ for 2 doses, then $4 \mathrm{mg} / \mathrm{kg}$ twice daily) and flucytosine with amphotericin B as agents with good penetration to the intraocular space (14). Fluconazole has been the preferred agent due to its safety and good ocular penetration and can be used solely with or without injection of antifungal agents into the intraocular space or vitrectomy (14). While vitrectomy is not considered to be essential, Riddel et al. strongly recommend the intravitreous injection of antifungal agents to achieve an adequate concentration in the intraocular space immediately (14).

The duration of systemic antifungal therapy is controversial, and there is no study defining a specific time period for which it should be given $(1,14)$. The Infectious Disease Society of America guidelines recommend treatment for at least four to six weeks until all ocular signs and symptoms subside, and Riddel et al. support this with the proposal for 
utilizing ophthalmologic examinations to determine the correct duration (14). We discontinued systemic antifungal treatment after four weeks with the improvement in the ocular findings, consistent with these guidelines.

In the present patient, vitrectomy coupled with systemic antifungal agent use was able to effectively treat the candida endophthalmitis with a careful ophthalmologic follow-up. Repeat ophthalmologic follow-up is essential for the early detection of ophthalmologic complications in patients with candidemia as recommended by the Infectious Disease Society of America.

The present middle-aged woman with newly diagnosed type 2 diabetes mellitus had candidemia from a retrograde UTI, which was then complicated by unilateral endophthalmitis. In patients with diabetes, the possibility of developing candidemia should always be considered, especially in patients with candiduria and symptoms of a UTI. If such patients are found to have candidemia, then frequent ophthalmologic examinations are crucial, as well as the adequate use of antifungal agents with or without surgical intervention. Above all, it is most important that when primarycare physicians encounter patients with an infection of unknown etiology, they search for the underlying cause before simply starting antibiotic therapy.

The authors state that they have no Conflict of Interest (COI).

\section{References}

1. Pappas PG, Kauffman CA, Andes D, et al. Clinical practice guidelines for the management of candidiasis: 2009 Update by the Infectious Diseases Society of America. Clin Infect Dis 48: 503-535, 2009.
2. Kauffman CA. Epidemiology and pathogenesis of candidemia in adults. In: UpToDate [Internet]. Post TW, Ed. UpToDate, Waltham. [cited 2014 Dec. 1]. Available from: http://www.uptodat e.com/home

3. Bukhary ZA. Candiduria; A review of clinical significance and management. Saudi J Kidney Dis Transplant 19: 350-360, 2008.

4. Kauffman CA. Candida infections of the bladder and kidneys. In: UpToDate [Internet]. Post TW, Ed. UpToDate, Waltham, 2014. [cited 2014 Dec. 1]. Available from: http://www.uptodate.com/home

5. Fisher JF, Kavanagh K, Sobel JD, Kauffman CA, Newman CA. Candida urinary tract infection: pathogenesis. Clin Infect Dis 52: S437-S451, 2011.

6. Abaitua $\mathrm{F}$, Rementerìa $\mathrm{A}$, Millan RS, et al. In vitro survival and germination of Candida albicans in the presence of nitrogen compounds. Microbiology 145: 1641-1647, 1999.

7. Frassetto L. Treatment and prevention of kidney stones: An update. Am Fam Physician 84: 1234-1242, 2011.

8. Pfaller M, Neofytos D, Diekema D, et al. Epidemiology and outcomes of candidemia in 3648 patients: data from the prospective antifungal therapy (PATH Alliance ${ }^{\circledR}$ ) registry, 2004-2008. Diagn Micr Infec Dis 74: 323-331, 2012.

9. Pappas P, Rex J, Lee J, et al. A prospective observational study of candidemia: Epidemiology, therapy, and influences on mortality in hospitalized adult and pediatric patients. Clin Infect Dis 37: 634643, 2003.

10. Ronald A, Ludwig E. Urinary tract infections in adults with diabetes. Int J Antimicrob Agents 17: 287-292, 2001.

11. Fisher JF, Sobel JD, Kauffman CA, Newman CA. Candida urinary tract infections - Treatment. Clin Infect Dis 52: S457-S466, 2011.

12. Jackson TL, Eykyn SJ, Graham EM, Stanford MR. Endogenous bacterial endophthalmitis: A 17-year prospective series and review of 267 reported cases. Surv Ophthalmol 48: 403-423, 2003.

13. Lingappan A, Wykoff CC, Albini TA, et al. Endogenous fungal endophthalmitis: Causative organisms, management strategies, and visual acuity outcomes. Am J Ophthlmol 153: 162-166, 2012.

14. Riddel J 4th, Comer GM, Kauffman CA. Treatment of endogenous fungal endophthalmitis: Focus on new antifungal agents. Clin Infect Dis 52: 648-653, 2011.

\footnotetext{
(C) 2015 The Japanese Society of Internal Medicine http://www.naika.or.jp/imonline/index.html
} 